\title{
Clinical Assessment of Emission- and Segmentation-Based MR-Guided Attenuation Correction in Whole-Body Time-of-Flight PET/MR Imaging
}

\author{
Abolfazl Mehranian ${ }^{1}$ and Habib Zaidi ${ }^{1-3}$ \\ ${ }^{I}$ Division of Nuclear Medicine and Molecular Imaging, Geneva University Hospital, Geneva, Switzerland; ${ }^{2}$ Geneva Neuroscience \\ Centre, University of Geneva, Geneva, Switzerland; and ${ }^{3}$ Department of Nuclear Medicine and Molecular Imaging, University of \\ Groningen, Groningen, The Netherlands
}

\begin{abstract}
The joint maximum-likelihood reconstruction of activity and attenuation (MLAA) for emission-based attenuation correction has regained attention since the advent of time-of-flight PET/MR imaging. Recently, we improved the performance of the MLAA algorithm using an MR imaging-constrained gaussian mixture model (GMM). In this study, we compare the performance of our proposed algorithm with standard 4-class MR-based attenuation correction (MRAC) implemented on commercial systems. Methods: Five head and neck ${ }^{18} \mathrm{~F}-\mathrm{FDG}$ patients were scanned on PET/MR imaging and PET/CT scanners. Dixon fat and water MR images were registered to CT images. MRAC maps were derived by segmenting the MR images into 4 tissue classes and assigning predefined attenuation coefficients. For MLAA-GMM, MR images were segmented into known tissue classes, including fat, soft tissue, lung, background air, and an unknown MR low-intensity class encompassing cortical bones, air cavities, and metal artifacts. A coregistered bone probability map was also included in the unknown tissue class. Finally, the GMM prior was constrained over known tissue classes of attenuation maps using unimodal gaussians parameterized over a patient population. Results: The results showed that the MLAA-GMM algorithm outperformed the MRAC method by differentiating bones from air gaps and providing more accurate patient-specific attenuation coefficients of soft tissue and lungs. It was found that the MRAC and MLAA-GMM methods resulted in average standardized uptake value errors of $-5.4 \%$ and $-3.5 \%$ in the lungs, $-7.4 \%$ and $-5.0 \%$ in soft tissues/lesions, and $-18.4 \%$ and $-10.2 \%$ in bones, respectively. Conclusion: The proposed MLAA algorithm is promising for accurate derivation of attenuation maps on time-of-flight PET/MR systems.
\end{abstract}

Key Words: PET/MRl; time-of-flight; attenuation correction; quantification; whole-body imaging

J Nucl Med 2015; 56:877-883

DOI: $10.2967 /$ jnumed.115.154807

$\mathbf{H}$ The tunities for enhancing the diagnostic confidence of PET and MR

Received Jan. 23, 2015; revision accepted Mar. 23, 2015.

For correspondence or reprints contact: Habib Zaidi, Geneva University Hospital, Division of Nuclear Medicine and Molecular Imaging, $\mathrm{CH}-1211$ Geneva, Switzerland.

E-mail:habib.zaidi@hcuge.ch

Published online Apr. 9, 2015.

COPYRIGHT (C 2015 by the Society of Nuclear Medicine and Molecular Imaging, Inc. imaging findings through the fusion of complementary structural and molecular information (1). The potential of PET/MR imaging in establishing a new multiparametric imaging paradigm has been a driving force for developing innovative solutions to tackle the challenges of these dual-modality systems.

Accurate attenuation correction (AC) of PET data is one of the major challenges of quantitative PET/MR imaging (2). In these systems, attenuation maps at $511 \mathrm{keV}$ should ideally be derived from the acquired MR images. However, in contrast to CT, MR imaging signals are not correlated with electron density and photon-attenuating properties of tissues but rather to proton density and magnetic relaxation properties. Therefore, there is no unique global mapping technique to convert MR imaging intensities to attenuation coefficients. In addition, lung tissues and cortical bones, which are 2 important tissue types in attenuation maps, exhibit low signals on images acquired using conventional MR pulse sequences because of their low water content and short transverse relaxation time. Therefore, the lungs, bones, and air pockets, which also produce a low signal, cannot be well differentiated from each other for the generation of MR imaging segmentationbased attenuation maps. Ultrashort-echo-time (UTE) and zeroecho-time (ZTE) MR pulse sequences have been investigated for the detection and visualization of bones as well as lung parenchyma (3-5). However, UTE/ZTE MR imaging is time-consuming and sensitive to magnetic field inhomogeneities and, as such, is not yet clinically feasible for whole-body MR imaging-guided AC (MRAC) of PET data. Current commercial PET/MR imaging systems use MR imaging segmentation-based approaches as the standard AC method. In these methods, MR images are segmented into 3 or 4 tissue classes-that is, background air, lung, and fat and nonfat soft tissues-and predefined constant attenuation coefficients are assigned to each tissue class $(6,7)$. However, inter- and intrapatient heterogeneity of attenuation coefficients in the different tissue classes is ignored by these approaches. Moreover, because bones and air cavities cannot be well discriminated in conventional MR sequences, these tissue classes are often replaced by soft tissue, which can lead to significant bias in PET tracer uptake quantification in different organs $(8,9)$. Hence, other $\mathrm{AC}$ techniques based on atlas registration, external transmission sources, and PET emission data have been explored and revisited in PET/MR imaging.

In atlas registration-based approaches, coregistered MR-CT atlas datasets are used to derive a pseudo-CT image from the patient's MR image or to learn a mapping function that predicts a pseudo-CT image $(10,11)$. These methods can solve the MRAC problem, particularly in brain imaging, provided that a perfect registration between the atlas and different patients can be achieved. However, such a registration is rarely possible in whole-body PET/MR imaging, 
because of substantial anatomic differences between patients and the limitations of registration algorithms. With advances in PET detector technology, time-of-flight (TOF) PET capability has been recently introduced in clinical PET/CT and PET/MR imaging scanners with the aim of improving lesion detectability and image quality as well as reducing acquisition time and the administrated activity of radiopharmaceuticals (12). After the recent rebirth of TOF PET, transmission- and emission-based methods have been revisited for deriving patient-specific attenuation maps in PET/MR imaging, thus potentially circumventing the uncertainties and obstacles of both standard and UTE/ZTE MRAC methods. In transmission-based approaches, attenuation coefficients are directly measured using external positron-emitting sources (13) or background radiation of lutetium oxyorthosilicate crystals (14) in a simultaneous transmission and emission acquisition mode. TOF information is then used to separate transmission from emission data. The limited timing resolution of current TOF PET scanners, however, does not allow for perfect separation of transmission data from emission data. Therefore, the calculated attenuation coefficients might be nonuniformly scaled and different from their expected values (13). In contrast, emission-based approaches rely only on emission data for joint maximum-likelihood estimation of activity and attenuation maps (MLAA) $(15,16)$. In fact, recent studies have demonstrated that both activity and attenuation distributions can be determined from TOF emission data, up to an unknown scaling factor $(16,17)$.

Emission-based AC methods are promising in TOF PET/MR imaging, where MR imaging anatomic information can be exploited to guide the estimation of the attenuation map. With the advent of sequential TOF PET/MR imaging systems, Salomon et al. (18) used both TOF and MR imaging anatomic information to constrain the MLAA algorithm. In their approach, MR images are segmented into many regions over which the attenuation coefficients are iteratively estimated from the emission data. Despite the fact that this approach substantially reduces noise and cross-talk artifacts between activity and attenuation maps, the reconstructed attenuation maps suffer from mis-segmentation errors, and the quantitative performance of the algorithm depends on the accurate correction of the scaling problem. We recently proposed an approach to use MR imaging spatial and CT statistical information in the joint estimation of activity and attenuation using a constrained gaussian mixture model (GMM) (19). In contrast to Salomon's method, MR images are segmented into a few tissue classes and incorporated into the GMM model. This approach allows the derivation of continuous attenuation maps with noise suppression, cross-talk, and the scale problem. In this work, we evaluated the performance of the proposed MLAA-GMM algorithm with standard 4-class MRAC over a patient population and demonstrated the potential advantage of MR imagingguided emission-based AC methods over conventional MR imagingguided segmentation-based approaches.

\section{MATERIALS AND METHODS}

\section{PET/MR Imaging and PET/CT Clinical Data Acquisition}

In this retrospective clinical study, 5 patients with head and neck carcinoma were imaged on the Philips Ingenuity TF PET/MR imaging and the Siemens Biograph mCT flow PET/CT scanners as part of the clinical workup. The average age and body mass index of patients were $57 \pm 5 \mathrm{y}$ and $24.6 \pm 5.1 \mathrm{~kg} / \mathrm{m}^{2}$, respectively. The patients were injected with an average of $271 \pm 9.3 \mathrm{MBq}$ of ${ }^{18} \mathrm{~F}-\mathrm{FDG}$ and after a standard uptake time of $60 \mathrm{~min}$, during which various MR sequences were acquired, underwent whole-body PET/MR imaging in an arms-down position. After an interval of 10-20 min, the patients underwent complementary PET/CT imaging of 10-12 min, also in the arms-down position. MR imaging was performed on the Achieva 3-T MR imaging subsystem of the PET/MR imaging scanner. A whole-body scan was acquired in shallow-breathing mode using a 3-dimensional multiecho fast field echo Dixon technique with the following parameters: repetition time of $5.7 \mathrm{~ms}$; echo time 1/echo time 2 of 1.45 and 2.6, respectively; flip angle of $10^{\circ}$, slice thickness of 3 $\mathrm{mm}$, and matrix size of $680 \times 680$. PET/CT scanning was performed in continuous-bed-motion mode with a bed speed of $1.1 \mathrm{~mm} / \mathrm{s}$, equivalent to 3 min per bed position in step-and-shoot mode. For CT-based AC (CTAC) of PET data, a multislice CT scan protocol was performed using the following parameters: $100-120 \mathrm{kVp}, 150 \mathrm{mAs}$, and 5-mm slice thickness. Supplemental Figure 1 (supplemental materials are available at http://jnm. snmjournals.org) shows a flowchart of the acquisition protocol used in this study. The TOF PET data of the mCT scanner was used for joint reconstruction of activity and attenuation maps, and the anatomic MR images acquired on the Ingenuity PET/MR imaging scanner were used for MR imaging-guided MLAA and 4-class MRAC methods. In-phase MR images were deformably registered to CT images using the Elastix software (20), with 5-level multiresolution registration and Mattes mutual information criterion. The resulting transformation fields were then used for registration of fat and water images.

\section{Attenuation Map Generation}

MR Imaging-Guided Emission-Based Attenuation Map. In the framework of the maximum-likelihood estimation, the MLAA algorithm jointly estimates activity $(\lambda)$ and attenuation $(\mu)$ maps by maximization of the Poisson log-likelihood of TOF PET emission data, that is,

$$
\begin{aligned}
(\hat{\lambda}, \hat{\mu})=\underset{\lambda \geq 0, \mu \geq 0}{\operatorname{argmax}}\{ & \sum_{i t} g_{i t} \log \left(n_{i} a_{i} \sum_{j} c_{i j t} \lambda_{j}+\bar{b}_{i t}\right) \\
& \left.-n_{i} a_{i} \sum_{j} c_{i j t} \lambda_{j}+\bar{b}_{i t}\right\},
\end{aligned}
$$

where $g_{i t}$ is the number of prompt coincidences measured by the PET scanner along the line-of-response $i$ in TOF bin $t, \bar{b}_{i t}$ is the expected number of random and scattered coincidences, $n_{i}$ is detector normalization factors, $a_{i}=\exp \left(-\sum_{j} l_{i j} \mu_{j}\right)$ is attenuation factors, $c_{i j t}$ is the geometric probability detection of annihilation events emitted from voxel $j$ along the line of response $i$ in TOF bin $t$, and $l_{i j}$ is the intersection length of the line of response $i$ with voxel $j$ in millimeters. Because the activity and attenuation variables are coupled in Equation 1, the MLAA algorithm follows an iterative alternating maximization approach (16). In this approach, the algorithm alternates between an emission and a transmission maximum-likelihood image reconstruction problem, which are, respectively, solved by a TOF ordinary Poisson ordered-subset expectation maximization (OSEM) algorithm and a non-TOF ordered-subset maximum-likelihood for transmission tomography (OS-MLTR) algorithm. In the proposed MLAA-GMM algorithm, we used a transmission maximum a posteriori image reconstruction for estimation of attenuation by exploiting a Markov random field smoothness function $\left(R_{M R F}\right)$ and a mixture of gaussian model $\left(R_{G M M}\right)$, defined as follows:

$$
\begin{gathered}
R_{M R F}(\mu)=-\beta \sum_{j} \sum_{k \in N_{j}} \frac{\omega_{j k}}{2}\left(\mu_{k}-\mu_{j}\right)^{2} \quad \text { Eq. } 2 \\
R_{G M M}(\mu)=\gamma \sum_{j} \log \left\{\sum_{h=1}^{H} \frac{\rho_{h}}{\sqrt{2 \pi} \sigma_{h}} \exp \left(-\frac{1}{2}\left(\frac{\mu_{j}-\bar{\mu}_{h}}{\sigma_{h}}\right)^{2}\right)\right\} .
\end{gathered}
$$

$R_{M R F}$ favors attenuation maps that are smooth based on the weighted ( $\omega)$ differences between voxel $j$ and its neighboring voxels in the 


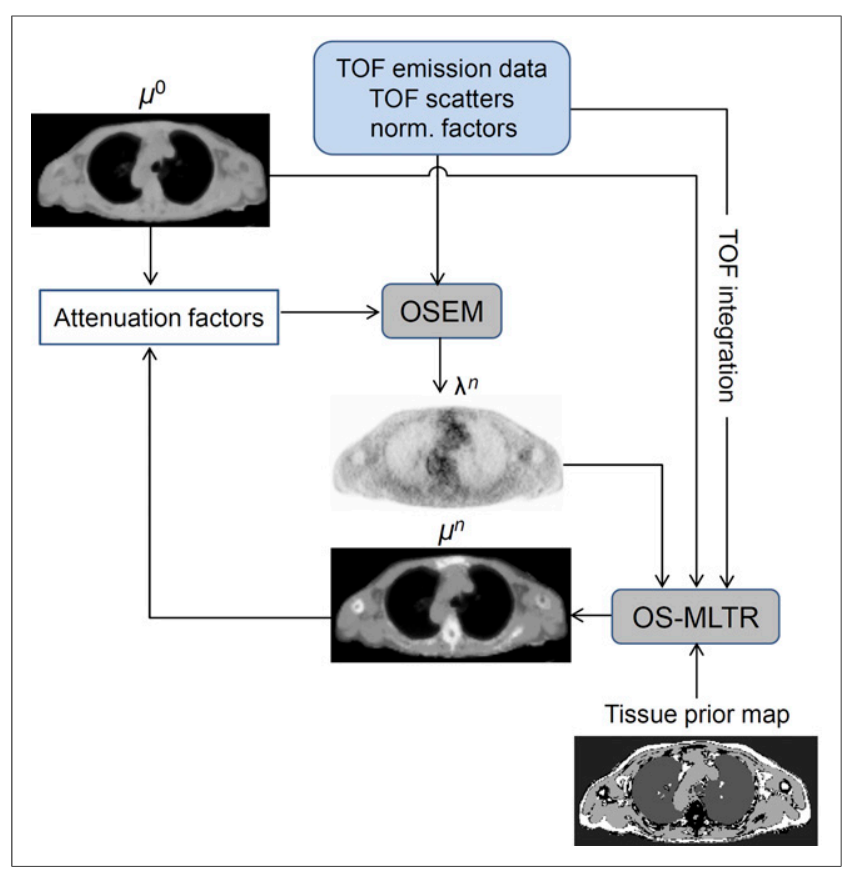

FIGURE 1. Flowchart of MLAA-GMM algorithm, which alternates between OSEM activity reconstruction and OS-MLTR attenuation reconstruction. Algorithm is initialized by 4-class MRAC attenuation map and MR imaging-based scatter sinograms. GMM model is spatially constrained by tissue prior map. norm. $=$ normalization.

neighborhood $N_{j} . R_{G M M}$ models the statistical distribution (histogram) of linear attenuation coefficients (LACs) in attenuation maps at $511 \mathrm{keV}$ as a mixture of $H$ known gaussian functions with mean $\bar{\mu}_{h}, \operatorname{SD} \sigma_{h}$, and mixture proportion $\rho_{h}(21)$. The parameters $\beta$ and $\gamma$ weight the impact of the penalty functions. In the proposed MLAA-GMM algorithm, the $R_{G M M}$ is iteratively approximated using a convex surrogate and spatially constrained by MR imaging anatomic information using a tissue prior map. This prior map contains known and unknown tissue classes, over which unimodal gaussians and a mixture of gaussians are, respectively,

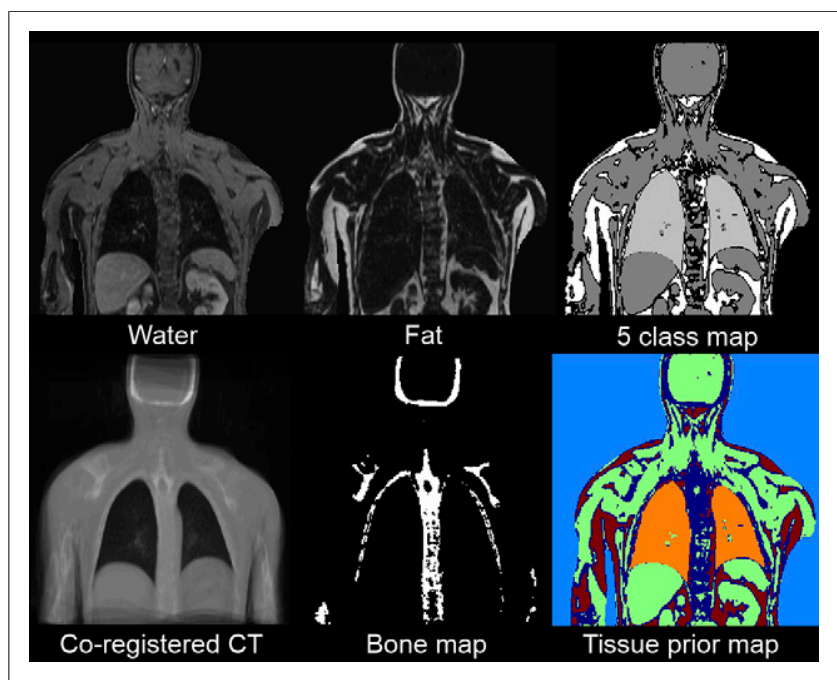

FIGURE 2. Derivation of tissue prior map. Fat and water Dixon images are segmented into background air, fat, soft tissue, lungs, and low-intensity class (5-class). Possible position of bones is determined from coregistered average CT image. imposed on the estimation of LACs during the OS-MLTR algorithm. Figure 1 presents the flowchart of the proposed MLAA-GMM algorithm. In this work, the algorithm was initialized by a uniform activity map and a 4-class MRAC map.

For generation of the tissue prior map (Fig. 2), Dixon water and fat MR images were segmented into 4 known tissue classes including fat, soft tissue, lungs, and background air and an additional unknown tissue class corresponding to the regions with low MR intensity (i.e., cortical bones, air pockets, and metal-induced susceptibility artifacts). Because of the partial-volume averaging and incomplete Dixon water and fat separation, spongy bones might possess moderate MR intensities in water images and therefore be classified as known soft and fat tissue classes instead of the unknown class. To eliminate this misclassification, we subtracted the fat from water images and used a bone probability map, obtained from a coregistered average CT, to ensure the inclusion of bones into the unknown class. The soft-tissue class was segmented by thresholding the fat-suppressed water images. The fat class was defined on the basis of the voxels of the fat image whose intensities are 50\% larger than the water image. The lungs and background air were segmented from in-phase MR images using a supervised seeded region-growing method implemented in the ITKSNAP software (22). In the proposed MLAA method, a mixture of 4 gaussians representing inside air, fat/soft mixture, and bone was used to guide the attenuation estimation over the unknown tissue class. The parameters of the mixture model and unimodal gaussians were estimated from 10 whole-body CTAC maps (19). Supplemental Figure 2 presents the estimated parameters.

MR Imaging-Guided Segmentation-Based Attenuation Map. Standard 4-class MRAC maps were derived by segmenting the Dixon water and fat images into 4 tissue classes: background air, lung, fat, and nonfat soft tissues. The background air, lungs, and fat tissue classes were obtained with the same procedure used for deriving the tissue prior map. The nonfat soft-tissue class was then defined as the complement of the segmented classes. In this procedure, all bones, air pockets, and susceptibility artifacts are assigned to the nonfat softtissue class. Mean attenuation coefficients of $0,0.0224,0.0864$, and $0.0975 \mathrm{~cm}^{-1}$ were assigned to background air, lungs, fat, and nonfat soft-tissue classes, respectively. The CT bed attenuation map was also added to the resulting MRAC maps.

CT-Based Attenuation Map. For comparison of the MLAA and MRAC methods against a reference AC method, CT-based attenuation maps were generated for each patient using Siemens e7 tools. High-resolution



FIGURE 3. Comparison of 4-class MRAC and MLAA-GMM attenuation maps with their reference CTAC map from representative ${ }^{18} \mathrm{~F}-\mathrm{FDG}$ $\mathrm{PET} / \mathrm{CT} / \mathrm{MR}$ imaging study. Display window is $0.08 \pm 0.055 \mathrm{~cm}^{-1}$. 




FIGURE 4. Close-up views of lungs in different displaying windows: $0.08 \pm 0.055 \mathrm{~cm}^{-1}$ (top) and $0.03 \pm 0.03 \mathrm{~cm}^{-1}$ (bottom).

CT images were downsampled to a resolution of $400 \times 400$. CT Hounsfield units were then converted to $511-\mathrm{keV}$ attenuation values using a $\mathrm{kVp}$ dependent bilinear mapping approach. The resulting attenuation maps were finally smoothed to the resolution of PET images using isotropic gaussian filter ( $4 \mathrm{~mm}$, in full width at half maximum).

\section{PET Image Reconstruction}

TOF PET data acquired on the $\mathrm{mCT}$ PET/CT scanner were reconstructed using a 3-dimensional TOF OSEM algorithm with 3 different AC methods: MR imaging-guided emission-based, MRguided segmentation-based, and CT-based. PET images were reconstructed with 3 iterations and 21 subsets and a matrix size of $200 \times 200$ with $4 \times 4 \times 2 \mathrm{~mm}$ voxels. For the MLAA-GMM algorithm, an inhouse software was developed for the native geometry of the $\mathrm{mCT}$ scanner with the following specifications: 400 radial bins, 168 azimuthal angles, 621 planes, and 13 TOF bins. The coincidence window width and effective TOF resolution of the scanner were 4.1 and 0.58 $\mathrm{ns}$, respectively. The activity and attenuation maps were reconstructed with 1 iteration and 2 subsets of the OSEM algorithm and 1 iteration and 3 subsets of the OS-MLTR algorithm with 20 global iterations. As mentioned above, the algorithm was initialized with a 4-class MRAC map with a TOF scatter simulated using the same MRAC map. On the basis of our previous work (19), the $\beta$ and $\gamma$ parameters (in Eqs. 2 and 3 ) were experimentally set to 80 and 0.015 , respectively. The estimated attenuation maps were then used for a standard OSEM PET image reconstruction.

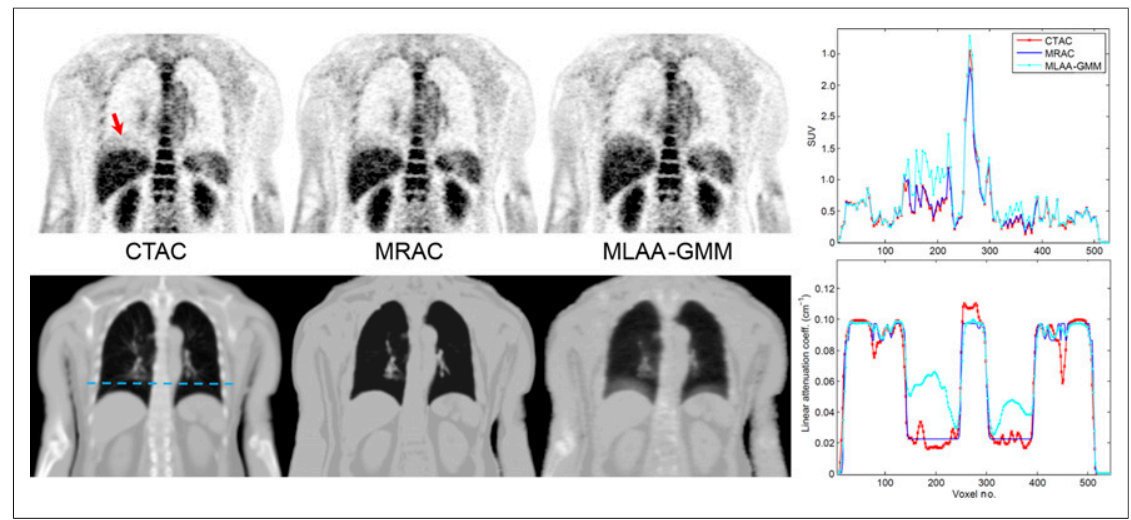

FIGURE 5. PET images and attenuation maps of patient presenting with respiratory-phase mismatch between PET and CT/MR imaging acquisitions. Activity and attenuation profiles along dashed line shown on CTAC attenuation map are also shown.

\section{Quantitative Evaluation}

The relative quantification error (bias) in the standardized uptake value (SUV) was calculated on a volume-of-interest (VOI) basis for each patient with respect to the reference CTAC PET as follows:

$$
\text { Bias }=100 \times \frac{\mathrm{SUV}_{m}-\mathrm{SUV}_{\mathrm{CTAC}}}{\mathrm{SUV}_{\mathrm{CTAC}}},
$$

where $m$ is the AC method used (MLAA or MRAC). For each patient, 14 VOIs were defined on normal tissue regions including the lungs (upper, middle, and lower portions of left and right lungs); aorta; liver; myocardium; thyroid; cerebrum; and fourth cervical (C4), third thoracic (T3), and fourth lumbar (L4) vertebra. VOIs were also defined on lesions localized on CTAC PET images. For the defined VOIs, the mean $(\mu)$, SD $(\sigma)$, and root-mean-squared error (RMSE) of bias $\left(\sqrt{\mu^{2}+\sigma^{2}}\right)$ was calculated. The correlation between $\operatorname{SUV}_{\text {MRAC }}$ and $\mathrm{SUV}_{\text {MLAA }}$ and reference SUV $_{\text {CTAC }}$ was determined using Pearson correlation analysis. The concordance between the SUVs was evaluated using Bland-Altman plots. The statistical differences in SUV bias was also calculated using the paired-sample $t$ test. The differences were considered statistically significant for a $P$ value of less than 0.05 .

\section{RESULTS}

Figure 3 compares the CTAC map of a representative patient with the attenuation maps obtained using the proposed MLAAGMM algorithm and the 4-class MRAC method. As shown, the MLAA-GMM algorithm can reasonably well estimate the attenuation coefficients of bones and discriminate air cavities, particularly paranasal sinuses. In contrast, the bones and air gaps in the MRAC maps are simply replaced by soft tissue, which can lead to quantification errors in the reconstructed PET images. Figure 4 shows the close-up views of the attenuation maps over the lungs in different displaying windows. The CTAC map shows that the lungs of this patient have a congested structure especially in the left lung. As indicated by the arrows, some of the condensed softtissue structures in the MRAC map have been erroneously segmented into the lung-tissue class, whereas the MLAA-GMM algorithm compensates for the mis-segmentation of these structures and also retrieves lung-density gradients. Because of respiratory motion of the lungs during PET acquisition and count-dependent performance of the MLAA algorithm, the estimated attenuation coefficients cannot preserve all local details. For this dataset, the lung attenuation coefficients of the CTAC, MLAA, and MRAC attenuation maps, filtered by a gaussian filter of $4 \mathrm{~mm}$ in full width at half maximum, were $0.0293 \pm 0.0077,0.0301 \pm 0.0075$, and $0.0225 \pm 0.0003 \mathrm{~cm}^{-1}$, respectively. Supplemental Figure 3 compares the attenuation maps of another patient study, where the MLAA-GMM algorithm also compensates for a mis-segmented pathology and accurately retrieves the lung-density gradient in a continuous fashion. Figure 5 shows activity and attenuation maps of another study in which the MLAA algorithm also compensated for respiratory-phase mismatch between activity and attenuation maps. As shown by the arrow, the uptake at the upper lobe of the liver has been underestimated by CTAC and MRAC methods because of undercorrection of attenuation. 
TABLE 1

Mean \pm SD of LACs of Different Tissue Classes of CTAC, MLAA, and MRAC Attenuation Maps Calculated over All Clinical Studies

\begin{tabular}{ccccc}
\hline Method & Lung & Fat & Soft tissue & Bone \\
\hline CTAC & $0.025 \pm 0.009$ & $0.087 \pm 0.009$ & $0.098 \pm 0.008$ & $0.118 \pm 0.012$ \\
MLAA & $0.027 \pm 0.008$ & $0.086 \pm 0.010$ & $0.097 \pm 0.006$ & $0.104 \pm 0.012$ \\
MRAC & $0.022 \pm 0.001$ & $0.086 \pm 0.004$ & $0.097 \pm 0.001$ & $0.095 \pm 0.005$ \\
\hline
\end{tabular}

As shown on the attenuation maps and profiles, the MLAA algorithm estimates correctly the underlying attenuation experienced by the emission data and therefore increases liver uptake, thus improving the detection and quantification of possible upper-lobe lesions.

Table 1 compares the mean \pm SD of LACs of different tissue classes of the CTAC, MLAA, and MRAC attenuation maps calculated over all patient datasets. For these classwise calculations, the lung, fat, and soft-tissue classes were obtained from the tissue prior map built for each patient (Fig. 2). The bone class was derived by thresholding CTAC images at $0.109 \mathrm{~cm}^{-1}$. As can be seen, the main difference between MLAA and MRAC methods sits over the lungs and bones, for which the maximum PET quantification errors occur when using standard MRAC methods. The results show that the MLAA-GMM algorithm outperforms the MRAC method over these tissue classes by estimating mean and SD of LACs, which are closer to those of the reference CTAC method. The proposed method, however, slightly over- and underestimates the mean of the LACs of lungs and bones, respectively. In fat and soft-tissue classes, both MLAA and MRAC attenuation maps have a mean similar to the CTAC maps, whereas the SD of only MLAA's LACs are close to those of CTAC maps.

The quantitative PET performance of MR imaging-guided AC methods was further evaluated in comparison with the CTAC method using VOI-based analysis. Table 2 summarizes the mean, $\mathrm{SD}$, and RMSE of the mean SUV ( $\left.\mathrm{SUV}_{\text {mean }}\right)$ bias in VOIs defined in normal tissue and lesions. Figure 6A shows the errors in each VOI, grouped for the lung and soft-tissue organs, whereas Figure $6 \mathrm{~B}$ shows the results for VOIs defined on bones or soft tissues

TABLE 2

Quantification Bias of PET MRAC and PET MLAA in Different Tissues with Respect to Reference PET CTAC

\begin{tabular}{lccc}
\hline \multicolumn{1}{c}{ VOI } & MRAC & MLAA & $P$ \\
\hline Lung & $-5.4 \pm 12.0(13.1)$ & $-3.5 \pm 6.6(7.5)$ & 0.1605 \\
\hline Aorta & $-9.5 \pm 10.5(14.1)$ & $-7.6 \pm 9.3(12.1)$ & 0.0942 \\
\hline Liver & $-7.4 \pm 1.8(7.6)$ & $-5.4 \pm 3.2(6.3)$ & 0.0376 \\
\hline Myocardium & $-9.2 \pm 6.0(11.0)$ & $-3.1 \pm 6.8(7.5)$ & 0.0027 \\
\hline Thyroid & $-1.9 \pm 8.8(9.0)$ & $-3.6 \pm 5.1(6.3)$ & 0.6574 \\
\hline Lesions & $-9.0 \pm 5.4(10.5)$ & $-4.5 \pm 5.3(7.0)$ & 0.0237 \\
Cerebrum & $-18.5 \pm 11.3(21.6)$ & $-11.6 \pm 6.0(13.1)$ & 0.0503 \\
C4 & $-22.9 \pm 2.7(23.1)$ & $-12.3 \pm 3.1(12.7)$ & 0.0011 \\
T3 & $-19.8 \pm 8.4(21.5)$ & $-12.6 \pm 8.6(15.2)$ & $<0.001$ \\
L4 & $-12.4 \pm 4.5(13.2)$ & $-4.2 \pm 4.4(6.1)$ & $<0.001$ \\
\multicolumn{4}{l}{ Data are mean \pm SD, with RMSE (\%) in parentheses. } \\
\hline
\end{tabular}

located close to bones. In this figure, the markers show the mean of bias in each VOI, whereas the horizontal bars and vertical boxes indicate the mean and 2 SDs of the bias between VOIs in each region. The results show that the MLAA-GMM algorithm generally gives rise to a reduced RMSE bias over all regions. For VOIs defined in the lungs $(n=30)$, the MRAC method underestimates $\mathrm{SUV}_{\text {mean }}$ by $-5.4 \% \pm 12.0 \%$, with an RMSE of $13.1 \%$, whereas MLAA-GMM reduces the errors to $-3.5 \% \pm 6.6 \%$, with an RMSE of $7.5 \%$. For the total VOIs defined on the aorta, myocardium, liver, and thyroid ( $n=20)$, MRAC and MLAA-GMM methods resulted in average SUV errors of $-7.0 \% \pm 6.6 \%$ (9.6\% RMSE) and $-4.9 \% \pm 5.5 \%$ (7.4\% RMSE), respectively. Over the lesions $(n=11)$, which were mainly mediastinal lymphoma, the MLAA-GMM reduced the errors from $-9.0 \% \pm 5.4 \%$ with an RMSE of $10.5 \%$ to $-4.5 \% \pm 5.3 \%$ with an RMSE of $7.0 \%$. Finally, for all regions in or near bones $(n=20)$, the MRAC and MLAA algorithms resulted in an average SUV error of $-18.4 \% \pm 7.9 \%(20.0 \%$ RMSE) and $-10.2 \% \pm 6.5 \%(12.1 \%$ RMSE), respectively. The statistical analysis revealed that there is an overall significant difference $(P<0.05)$ between the proposed MLAA-GMM and MRAC methods in bones, malignant lesions, and most soft-tissue regions. The results also showed that the bias differences in the lungs are insignificant.

The SUV correlation and concordance of the MLAA and MRAC methods with reference CTAC was further evaluated. Figure 7 (top) shows the scatterplots of the $\mathrm{SUV}_{\text {mean }}$ in all studied VOIs between PET CTAC, PET MRAC, and PET MLAA with correlation and regression coefficients. The results show that PET MLAA and PET MRAC are highly correlated with PET CTAC, with $R^{2}$ values of 0.982 and 0.992 , respectively. Figure 7 (bottom) also shows the results of Bland-Altman concordance analysis. The limits of agreement were calculated from logarithmically transformed values. Differences and limits of agreement are expressed as a function of average SUVs. As shown by the regression lines of the difference, MRAC and MLAA AC methods result in a systematic underestimation of SUV by up to $9.85 \%$ and $6.75 \%$, respectively. However, MLAA clearly outperforms MRAC by reducing the errors and their dispersion.

\section{DISCUSSION}

Interest in the estimation of PET attenuation maps from emission data has recently been revived in the context of TOF PET/MR imaging AC to overcome the limitations and quantification errors of standard segmentation-based MRAC methods $(18,19)$. In this work, we compared the performance of our previously reported MLAA algorithm with the standard MRAC method to demonstrate the potential of emission-based AC methods in TOF PET/MR imaging.

In segmentation-based MRAC methods, the mis-segmentation of the lungs and the assignment of nonpatient-specific lung attenuation coefficients can result in PET quantification errors. 

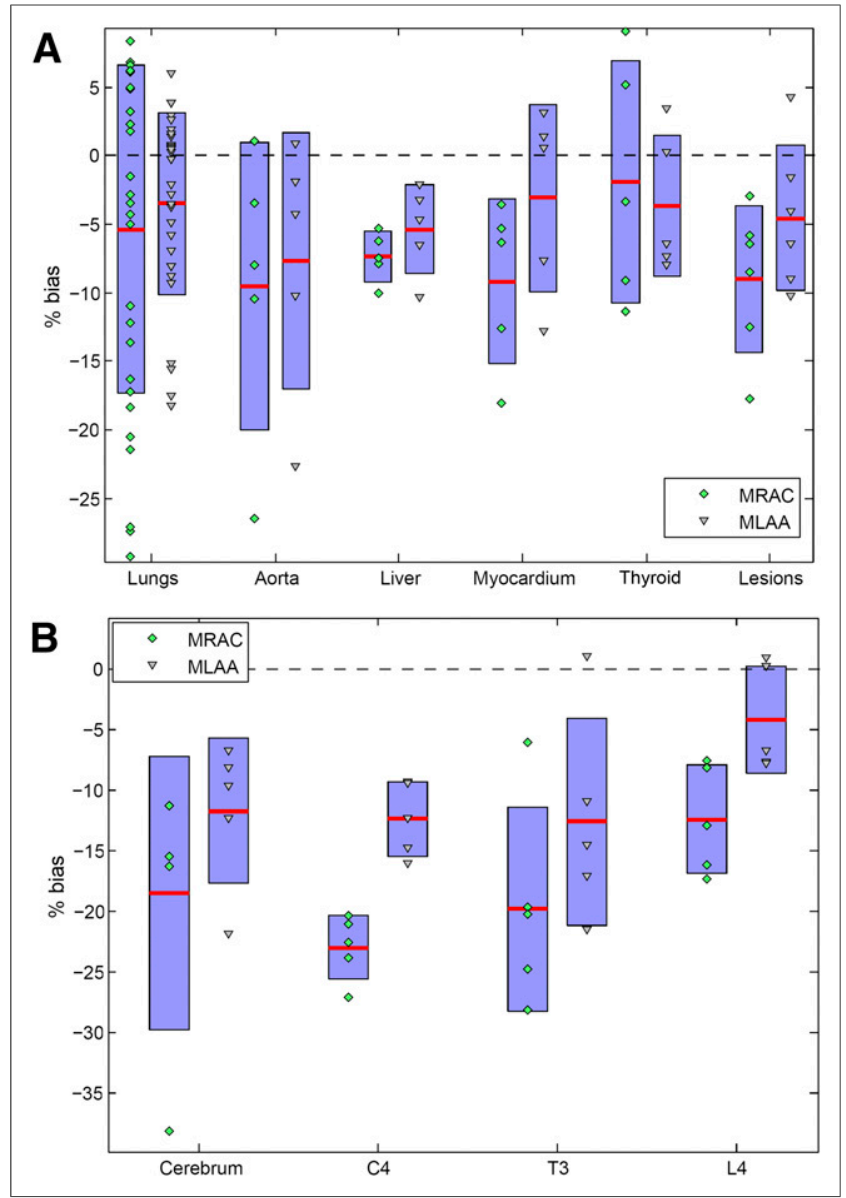

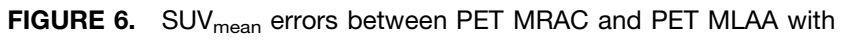
respect to reference PET CTAC images in VOls defined on normal tissues and lesions (A) and in/near bones (B). Means and SD are indicated by horizontal bars and vertical boxes, respectively. C4, T3, and L4 represent fourth cervical, third thoracic, and fourth lumbar vertebra.

Our results demonstrated that, consistent with previously reported results (23), the MRAC method results in errors in the lungs of up to $26 \%$ (Fig. 6). Because of their low proton density and short $\mathrm{T}_{2}{ }^{*}$ relaxation time, the lungs show low signal intensity in the fast gradient-recalled echo pulse sequences conventionally used in wholebody MR imaging acquisitions. Therefore, in conjunction with the limitations of segmentation-based MRAC algorithms, the soft-tissue structures of the lungs, for example, pulmonary vessels and bronchioles, might be mis-segmented into lung class. Our results showed that the MLAA-GMM algorithm not only fairly compensates for the mis-segmentations but also derives more patient-specific lung LACs (Fig. 4; Supplemental Fig. 3). Hence, as reported in Table 2, the RMSE in the lungs was reduced from $13.1 \%$ to $7.5 \%$. In contrast to Salomon et al. (18), our proposed MLAA estimates continuous attenuation coefficients and thus retrieves the lung-density gradients and intrapatient variability of lung attenuation coefficients. Continuous lung LACs can also be derived from atlas registration-based AC methods. However, these approaches had a limited success because of the low signal of the lungs (10).

Another limitation of segmentation-based MRAC is that the identification of bones, which produce low signal intensity in gradient-recalled echo MR imaging pulse sequences, is challenging. In 4-class MRAC maps, the bones are replaced by soft tissue, which on the basis of our results can lead to a mean bias of $-18.4 \%$. This mean bias is consistent with the greater than $15 \%$ errors reported previously $(9,23)$. As demonstrated in this work, the proposed MLAAGMM algorithm is capable of estimating bone attenuation coefficients, thus outperforming the MRAC method by reducing the mean error to $-10.2 \%$. Bezrukov et al. reported that a combination of segmentation and atlas registration-based AC methods can effectively reduce bone SUV errors of the 4-class MRAC from $-16.1 \%$ to $-4.7 \%$ (8). However, the performance of these techniques depends on accurate atlas registration and robust prediction of attenuation coefficients. In contrast, several CT images are registered to a patient's in-phase MR image in our MR imaging-constrained MLAA algorithm to only roughly indicate the position of the bones. Therefore, this technique is in principle not subjected to misregistration errors.

As indicated in Supplemental Figure 2, we set the means of gaussian models defined in known tissue classes to the mean LACs used in the 4-class MRAC maps. Therefore, for high values of the $\gamma$ parameter in Equation 3, the MLAA-GMM is essentially reduced to a 4-class MRAC method. The results show that the MLAA and MRAC methods present similar mean LACs in fat and soft-tissue classes (Table 1); however, the SDs (inter-/intravariability) of LACs in the MLAA attenuation maps are similar to those of reference CTAC maps. Combined with more accurate derivation of lung and bone LACs, our MLAA-GMM approach resulted in improved quantitative performance over soft-tissue organs, compared with the MRAC method (Table 2).

The results of this feasibility study demonstrate the potential of the emission-based AC methods for accurate AC in TOF PET/MR imaging. However, it is worth highlighting the limitations of the proposed algorithm and the study conducted herein. Similar to other maximum a posteriori reconstruction techniques, the performance of the MLAA-GMM depends on the selection of the regularization parameters, especially the $\gamma$ parameter of the GMM model. In this work, we set the $\gamma$ and the $\beta$ parameter in Equation 2 to experimentally optimized values for a few simulation and clinical studies (19). In general, the MLAA algorithm is time-consuming because it alternates between an emission and transmission tomographic reconstruction. The preparation of a tissue prior map can further increase the

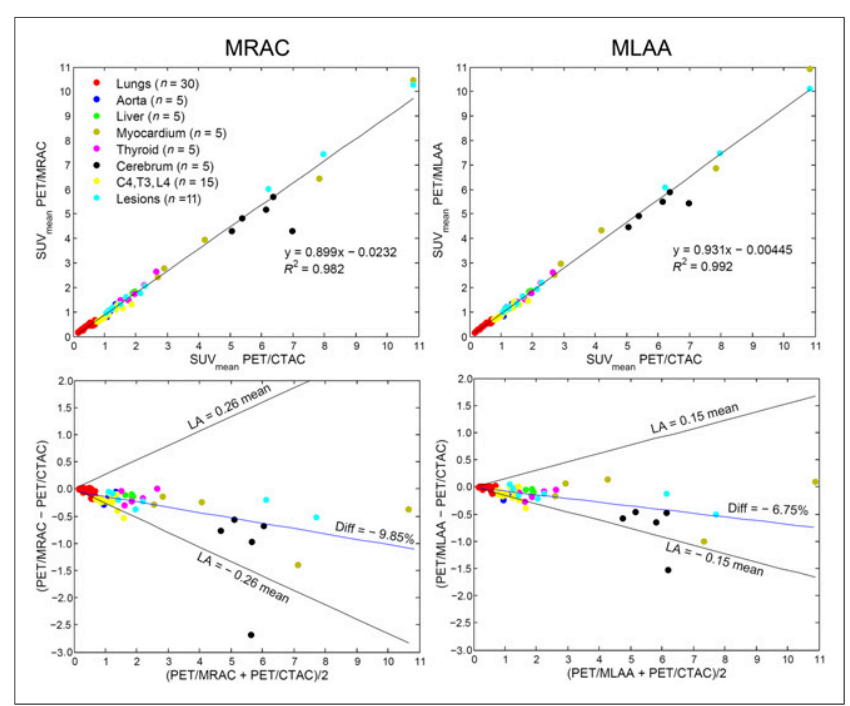

FIGURE 7. (Top) Scatterplots between $\mathrm{SUV}_{\text {mean }}$ of PET images reconstructed using CTAC, MLAA-GMM, and MRAC AC methods. (Bottom) $S U V_{\text {mean }}$ Bland-Altman concordance plots. 
computational burden of the proposed MLAA-GMM method. In this first clinical study, a relatively small number of PET/MR/CT datasets could be included. Therefore, the statistical significance of our results might be subject to a degree of uncertainty. Future work will focus on further evaluation of the studied AC methods using a large clinical patient database acquired with different tracers to pave the way for translation of emission-based AC methods into the clinic.

\section{CONCLUSION}

In this work, the performance of an MR imaging-guided emissionbased AC method was compared with the standard segmentationbased MRAC method using clinical studies. It was demonstrated that the proposed constrained MLAA algorithm is promising for deriving patient-specific attenuation maps, especially in the lungs and bones. Our results showed that the MRAC method resulted in average SUV errors of $-5 \%$ and $-18 \%$ in the lungs and bones, and the proposed algorithm reduced the errors to $-3 \%$ and $-10 \%$, respectively. Emission-based AC is promising in clinical TOF PET/MR imaging and presents the potential to replace conventional segmentation-based methods implemented on commercial systems.

\section{DISCLOSURE}

The costs of publication of this article were defrayed in part by the payment of page charges. Therefore, and solely to indicate this fact, this article is hereby marked "advertisement" in accordance with 18 USC section 1734. This work was supported in part by the Swiss National Science Foundation under grant SNSF 31003A-149957 and the Indo-Swiss Joint Research Programme ISJRP-138866. No other potential conflict of interest relevant to this article was reported.

\section{REFERENCES}

1. Torigian DA, Zaidi H, Kwee TC, et al. PET/MR imaging: technical aspects and potential clinical applications. Radiology. 2013;267:26-44.

2. Bezrukov I, Mantlik F, Schmidt H, Scholkopf B, Pichler BJ. MR-based PET attenuation correction for PET/MR imaging. Semin Nucl Med. 2013;43:45-59.

3. Keereman V, Fierens Y, Broux T, De Deene Y, Lonneux M, Vandenberghe S. MRI-based attenuation correction for PET/MRI using ultrashort echo time sequences. J Nucl Med. 2010;51:812-818.

4. Zeimpekis K, Delso G, Wiesinger F, Veit-Haibach P, von Schulthess G, Grimm R. Investigation of 3D UTE MRI for lung PET attenuation correction [abstract]. J Nucl Med. 2014;55(suppl 1):2103.
5. Delso G, Wiesinger F, Sacolick LI, et al. Clinical evaluation of zero-echo-time MR imaging for the segmentation of the skull. J Nucl Med. 2015;56:417-422.

6. Martinez-Möller A, Souvatzoglou M, Delso G, et al. Tissue classification as a potential approach for attenuation correction in whole-body PET/MRI: evaluation with PET/CT data. J Nucl Med. 2009;50:520-526.

7. Zaidi H, Ojha N, Morich M, et al. Design and performance evaluation of a wholebody Ingenuity TF PET-MRI system. Phys Med Biol. 2011;56:3091-3106.

8. Bezrukov I, Schmidt H, Mantlik F, et al. MR-based attenuation correction methods for improved PET quantification in lesions within bone and susceptibility artifact regions. J Nucl Med. 2013;54:1768-1774.

9. Arabi H, Rager O, Alem A, Varoquaux A, Becker M, Zaidi H. Clinical assessment of MR-guided 3-class and 4-class attenuation correction in PET/MR. Mol Imaging Biol. 2015;17:264-276.

10. Hofmann M, Steinke F, Scheel V, et al. MRI-based attenuation correction for PET/MRI: a novel approach combining pattern recognition and atlas registration. J Nucl Med. 2008;49:1875-1883.

11. Navalpakkam BK, Braun H, Kuwert T, Quick HH. Magnetic resonance-based attenuation correction for PET/MR hybrid imaging using continuous valued attenuation maps. Invest Radiol. 2013;48:323-332.

12. Surti S. Update on time-of-flight PET Imaging. J Nucl Med. 2015;56:98-105.

13. Mollet $P$, Keereman V, Bini J, Izquierdo-Garcia D, Fayad ZA, Vandenberghe S. Improvement of attenuation correction in time-of-flight PET/MR imaging with a positron-emitting source. J Nucl Med. 2014;55:329-336.

14. Rothfuss H, Panin V, Moor A, et al. LSO background radiation as a transmission source using time of flight. Phys Med Biol. 2014;59:5483-5500.

15. Nuyts J, Dupont P, Stroobants S, Benninck R, Mortelmans L, Suetens P. Simultaneous maximum a posteriori reconstruction of attenuation and activity distributions from emission sinograms. IEEE Trans Med Imaging. 1999;18:393-403.

16. Rezaei A, Defrise M, Bal G, et al. Simultaneous reconstruction of activity and attenuation in time-of-flight PET. IEEE Trans Med Imaging. 2012;31:22242233.

17. Defrise M, Rezaei A, Nuyts J. Time-of-flight PET data determine the attenuation sinogram up to a constant. Phys Med Biol. 2012;57:885-899.

18. Salomon A, Goedicke A, Schweizer B, Aach T, Schulz V. Simultaneous reconstruction of activity and attenuation for PET/MR. IEEE Trans Med Imaging. 2011;30:804-813.

19. Mehranian A, Zaidi H. Joint estimation of activity and attenuation in whole-body TOF PET/MRI using constrained gaussian mixture models. IEEE Trans Med Imaging. March 5, 2015 [Epub ahead of print].

20. Klein S, Staring M, Murphy K, Viergever MA, Pluim JP. Elastix: a toolbox for intensity-based medical image registration. IEEE Trans Med Imaging. 2010; 29:196-205.

21. Anderson JMM, Yoon-Chul K, Votaw JT. Concurrent segmentation and estimation of transmission images for attenuation correction in positron emission tomography. IEEE Trans Nucl Sci. 2009;56:136-146.

22. Yushkevich PA, Piven J, Hazlett HC, et al. User-guided 3D active contour segmentation of anatomical structures: significantly improved efficiency and reliability. Neuroimage. 2006;31:1116-1128.

23. Keereman V, Holen RV, Mollet P, Vandenberghe S. The effect of errors in segmented attenuation maps on PET quantification. Med Phys. 2011;38:6010-6019. 